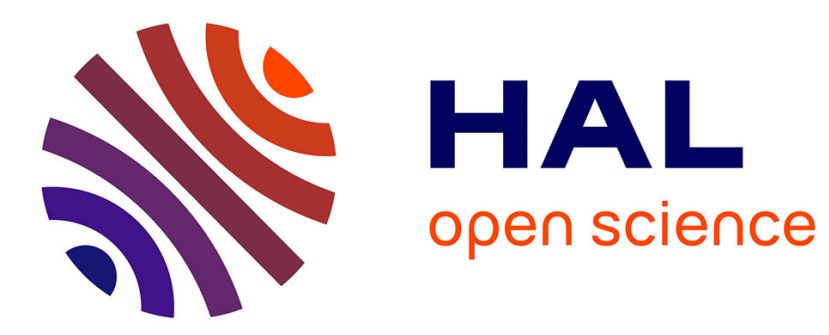

\title{
Blasius flow and heat transfer of fourth-grade fluid with slip
}

\author{
Bikash Sahoo, Sébastien Poncet
}

\section{To cite this version:}

Bikash Sahoo, Sébastien Poncet. Blasius flow and heat transfer of fourth-grade fluid with slip. Applied Mathematics and Mechanics, 2013, 34 (12), pp.1465-1480. 10.1007/s10483-013-1760-6 . hal00975631

\section{HAL Id: hal-00975631 \\ https://hal.science/hal-00975631}

Submitted on 10 Apr 2014

HAL is a multi-disciplinary open access archive for the deposit and dissemination of scientific research documents, whether they are published or not. The documents may come from teaching and research institutions in France or abroad, or from public or private research centers.
L'archive ouverte pluridisciplinaire HAL, est destinée au dépôt et à la diffusion de documents scientifiques de niveau recherche, publiés ou non, émanant des établissements d'enseignement et de recherche français ou étrangers, des laboratoires publics ou privés. 


\title{
Blasius flow and heat transfer of a fourth grade fluid with slip
}

\author{
Bikash Sahoo \\ Department of Mathematics, National Institute of Technology, Rourkela, Orissa, \\ India, \\ Tel.+916612462706, bikashsahoo@nitrkl.ac.in
}

Sébastien Poncet

Aix-Marseille Université, CNRS, Laboratoire M2P2 UMR 7340, 13451, Marseille, France,

Tel.+33491118555 - Fax +33491118502, sebastien.poncet@univ-amu.fr

\begin{abstract}
This investigation deals with the effects of slip, magnetic field and non-Newtonian flow parameters on flow and heat transfer of an incompressible, electrically conducting fourth grade fluid past an infinite porous plate subject to suction and blowing, known as the Blasius flow problem. The heat transfer analysis has been carried out for two heating processes, namely, when the plate is assumed to be at higher temperature than the fluid, and in the second case, the plate is assumed to be insulated. The order of the resulting differential equations exceeds the available adherence boundary conditions due to the presence of the higher order material derivatives of the strain tensor in the constitutive relation for the fourth grade fluid. The system of highly non-linear differential equations is solved using a fourth order Runge-Kutta method along with a shooting method for moderate values of the flow and heat transfer parameters. The effective Broyden's technique has been adopted in order to improve the initial guesses and to satisfy the boundary conditions at infinity. The combined effects of the non-Newtonian flow parameters and slip on the momentum and thermal boundary layers have been discussed in details. An exceptional crossovers is obtained in the velocity profile in presence of slip. The fourth grade fluid parameter is found to increase the momentum boundary layer thickness, whereas the slip parameter substantially decreases it. Similarly, the non-Newtonian fourth grade fluid parameters and the slip have opposite effects on the thermal boundary layer thickness.
\end{abstract}

Key words: Blasius flow, Partial slip, Fourth grade fluid, Broyden's method.

Preprint submitted to Appl. Math. Mech.




\section{Introduction}

Despite their applications in many industrial processes, the boundary layer flow of higher order fluid of differential type, namely the fourth grade fluid here, has drawn the attention of only few researchers during the last few decades. The main reason is that the constitutive equation of the fourth grade fluid is very complex involving a wide number of parameters. Moreover, the order of the differential equation corresponding to the momentum equation is exceptionally large, and exceeds the number of available boundary conditions, even in the simplest boundary layer flow past a flat plate. Renardy [1] has shown that such fluids are the last to have a stable state of rest. They have been considered in few other configurations such as plane Poiseuille $[2,3]$ or Couette [4] flows or recently, in an asymmetric channel [5].

The unsteady and steady flows of a fourth grade fluid, subject to a transverse uniform magnetic field past a porous plate has been investigated by Hayat et al. [6]. The boundary layer approximation resulted in a highly nonlinear differential equation of order six corresponding to the momentum equation with only two adherence boundary conditions. The finite difference method has been adopted to solve the sixth order nonlinear differential equation by augmenting four extra asymptotic boundary conditions. Hayat et al. [7] have studied the unsteady flow of a fourth grade fluid past a porous plate. They have successively reduced the order and number of independent variables of the resulting partial differential equations using Lie point symmetries. Subsequently, Sajid et al. [8] have studied the steady flow of a fourth grade fluid past a plate with suction and injection velocities at the surface of the plate. The homotopy analysis method (HAM) is used to find the solution of the fifth order highly nonlinear differential equation with two available boundary conditions. Hayat et al. [9] have used the HAM method to investigate the influence of the heat transfer on the flow of a fourth grade fluid past a porous plate. The unsteady MHD flow of a fourth grade fluid due to an oscillating plate with suction and blowing has been investigated numerically by Wang and $\mathrm{Wu}[10]$. The corresponding sixth order nonlinear partial differential equation has been solved by finite difference technique after augmenting four asymptotic boundary conditions. Recently, Marinca et al. [11] have used optimal homotopy asymptotic method to solve the resulting highly nonlinear momentum equation with no-slip boundary condition.

The foremost reason which motivated for the present study is the nonlinearity and the order of the differential equation corresponding to the momentum equation due to the flow of a fourth grade fluid. The order of the resulting differential equation exceeds drastically from the available adherence boundary conditions due to the presence of the higher order material derivatives of the strain tensor in the constitutive relation for the fourth grade fluid. The 
literature survey reveals that in the aforementioned studies, respective authors [6-10] have augmented additional boundary conditions at infinity. However, these are relatively weak and physically plausible. Few other interesting results from the literature survey, which arouse a curiosity to consider the MHD flow and heat transfer of a fourth grade fluid with partial slip boundary condition are as follows. Hayat et al. [6] have reported that in presence of the magnetic field, the boundary layer thickness decreases with an increase in the fourth grade fluid parameter, and the magnetic field has a substantial effect on the boundary layer thickness. The conclusions of the study of the steady flow of a fourth grade fluid past a porous plate by Sajid et al. [8] reveal that there does not exist a solution in the case of injection and the boundary layer thickness is rapidly decreased with an increase in the fourth grade fluid parameter for all values of the suction parameter exceeding one. Moreover, the recent study [9] of the flow and heat transfer of a fourth grade fluid past a porous plate depicts that the thermal boundary layer thickness increases with an increase in the fourth grade fluid parameter. We can cite also the recent contribution of Islam et al. [3], who investigated the steady flow of fourth-grade fluid in a infinite plane Poiseuille channel with slippage using the optimal HAM. They highlighted that this technique provides more satisfactory results than the homotopy perturbation technique.

Hayat et al. [12] considered partial slip effects on the flow and heat transfer characteristics of a third grade fluid past a porous plate using the homotopy analysis method. Nadeem et al. [13] studied the effects of partial slip on a fourth-grade fluid with variable viscosity contained in a stationary cylinder. Analytic solutions of velocity and temperature have been developed and discussed for the Reynolds and Vogels models. The present paper is the extension of the recent work of Sahoo [14], who investigated the flow and heat transfer of an electrically conducting third grade fluid past an infinite plate with partial slip. It deals in the present case with the numerical study of the slip flow and heat transfer of an incompressible, electrically conducting fourth grade fluid past an infinite porous plate subject to suction and blowing. The heat transfer analysis has been carried out for two heating processes, namely, when the plate is assumed to be at higher temperature than the fluid, and in the second case, the plate is assumed to be insulated. To the best of our knowledge, no attention has been given to the effects of partial slip on the MHD boundary layer flow and heat transfer of a fourth grade fluid over a flat plate. Even, the hydrodynamic boundary layer flow and heat transfer of a fourth grade fluid with slip boundary condition is not discussed so far. However, some non-Newtonian fluids like polymer melts exhibit macroscopic wall slip, which makes the no-slip condition fully inappropriate. It is noteworthy to point out that the quantitative behavior of higher grade fluids is strongly dependent on the choice of the material parameters. At the present time, there appears to be no experimental data determining these parameters, particularly the material parameters in the theory of fourth grade fluids. The present investigation is a 
step towards understanding the effects of the slip parameter on the flow and heat transfer of a fourth grade fluid.

This paper is divided as follows: in Section 2, the problem is mathematically formulated to get a system of ordinary differential equations with appropriate boundary conditions and is solved using an effective second order numerical scheme in Section 3. The effects of the flow parameters (partial slip, heat transfer, non newtonian parameters, suction/injection velocity) on a Blasius flow of a fourth grade fluid are discussed in details in Section 4 before some concluding remarks in Section 5 .

\section{Formulation of the problem}

We consider the steady two-dimensional flow of an incompressible fourth grade fluid past an infinite porous plate (infinite in the $x$ - direction), coinciding with the plane $y=0$. The flow far away from the plate is uniform, and is in the direction $x$ parallel to the plate. A transverse uniform magnetic field $\left(0, B_{0}, 0\right)$ (Fig. 1) has been applied at the surface of the plate and the surface admits partial slip.

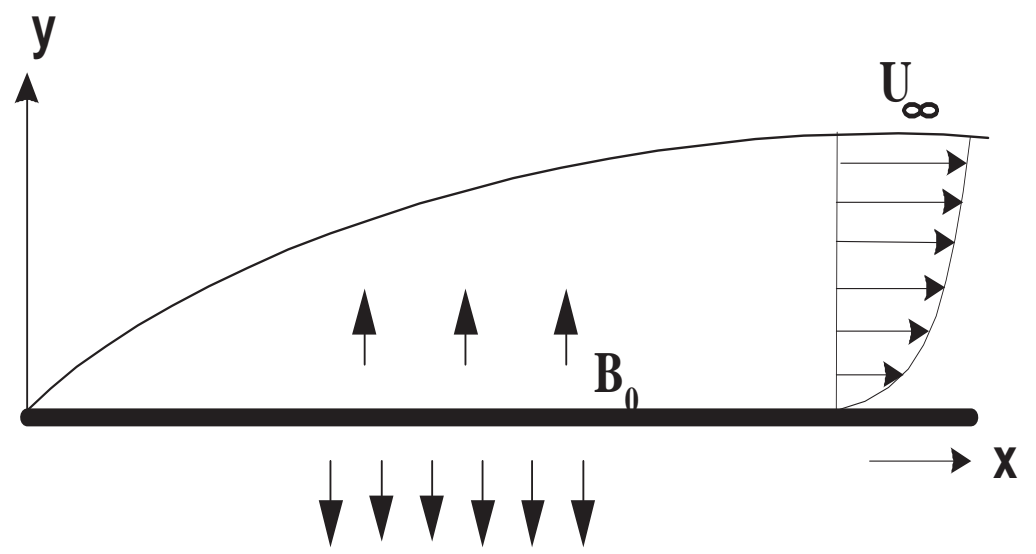

$\mathbf{V}_{0}$

Fig. 1. A sketch of the Blasius flow problem.

Thus, we seek velocity and temperature fields under the form:

$$
\begin{aligned}
& \mathbf{V}=u(y) \mathbf{i}+v(y) \mathbf{j} \\
& \theta=\theta(y)
\end{aligned}
$$

where $u$ and $v$ are the components of the velocity in the $x$ and $y$ directions respectively and $\theta$ denotes the temperature. 


\subsection{Flow analysis}

Neglecting the body force, the equations of motion and continuity for an electrically conducting, incompressible fluid write:

$$
\begin{gathered}
\rho \frac{d \mathbf{V}}{d t}=\nabla \cdot \mathbf{T}+\mathbf{J} \times \mathbf{B} \\
\nabla \cdot \mathbf{V}=0
\end{gathered}
$$

where $d / d t$ denotes the temporal material derivative, $\rho$ is the density of the fluid assumed to be constant, $\mathbf{T}$ is the Cauchy stress for an incompressible homogeneous fluid of grade four, and $\mathbf{J}$ is the electric current. The term due to the Lorentz force $(\mathbf{J} \times \mathbf{B})$ is simplified by making some assumptions valid for small magnetic Reynolds numbers and no displacement current [15]: the physical quantities are kept constant, the magnetic field is perpendicular to the velocity field, the induced magnetic field remains small compared to the applied magnetic one and the electrical field is assumed to be zero.

Under the consideration of the flow, it follows from (1) and (4) that:

$$
v=-V_{0}
$$

where $V_{0}>0$ is the suction velocity and $V_{0}<0$ is the injection velocity. $V_{0}$ is considered as being constant.

The Cauchy stress $\mathbf{T}$ for an incompressible fourth grade fluid has the constitutive equation (see in [5] for example):

$$
\begin{aligned}
\mathbf{T}= & -p \mathbf{I}+\mu \mathbf{A}_{\mathbf{1}}+\alpha_{1} \mathbf{A}_{\mathbf{2}}+\alpha_{2} \mathbf{A}_{\mathbf{1}}{ }^{2} \\
& +\beta_{1} \mathbf{A}_{\mathbf{3}}+\beta_{2}\left(\mathbf{A}_{\mathbf{2}} \mathbf{A}_{\mathbf{1}}+\mathbf{A}_{\mathbf{1}} \mathbf{A}_{\mathbf{2}}\right)+\beta_{3}\left(\operatorname{tr} \mathbf{A}_{\mathbf{1}}{ }^{2}\right) \mathbf{A}_{\mathbf{1}} \\
& +\gamma_{1} \mathbf{A}_{\mathbf{4}}+\gamma_{2}\left(\mathbf{A}_{\mathbf{3}} \mathbf{A}_{\mathbf{1}}+\mathbf{A}_{\mathbf{1}} \mathbf{A}_{\mathbf{3}}\right)+\gamma_{3} \mathbf{A}_{\mathbf{2}}{ }^{2}+\gamma_{4}\left(\mathbf{A}_{\mathbf{2}} \mathbf{A}_{\mathbf{1}}{ }^{2}+\mathbf{A}_{\mathbf{1}}{ }^{2} \mathbf{A}_{\mathbf{2}}\right) \\
& +\gamma_{5}\left(\operatorname{tr} \mathbf{A}_{\mathbf{2}}\right) \mathbf{A}_{\mathbf{2}}+\gamma_{6}\left(\operatorname{tr} \mathbf{A}_{\mathbf{2}}\right) \mathbf{A}_{\mathbf{1}}{ }^{2}+\left[\gamma_{7}\left(\operatorname{tr} \mathbf{A}_{\mathbf{3}}\right)+\gamma_{8} \operatorname{tr}\left(\mathbf{A}_{\mathbf{2}} \mathbf{A}_{\mathbf{1}}\right)\right] \mathbf{A}_{\mathbf{1}}
\end{aligned}
$$

where $\mathbf{I}$ is the identity tensor. The coefficient $\mu$ is the coefficient of viscosity $(\mathrm{kg} / \mathrm{m} / \mathrm{s}) . \alpha_{1}$ and $\alpha_{2}$ are the second order material constants $(\mathrm{kg} / \mathrm{m})$, $\beta_{1}, \ldots, \beta_{3}$ the third order material constants $(\mathrm{kg} . \mathrm{s} / \mathrm{m})$ and $\gamma_{1}, \ldots, \gamma_{8}$ the fourth order material constants $\left(\mathrm{kg} . \mathrm{s}^{2} / \mathrm{m}\right)$. The first to fourth order Rivlin-Ericksen tensors $\mathbf{A}_{\mathbf{i}}(i=1, \ldots, 4)$ are defined by the recursive relation: 


$$
\begin{aligned}
& \mathbf{A}_{\mathbf{0}}=\mathbf{I} \\
& \mathbf{A}_{\mathbf{n}}=\frac{d \mathbf{A}_{\mathbf{n}-\mathbf{1}}}{d t}+\mathbf{A}_{\mathbf{n}-\mathbf{1}} \nabla \mathbf{V}+(\nabla \mathbf{V})^{T} \mathbf{A}_{\mathbf{n}-\mathbf{1}}, n \geq 1
\end{aligned}
$$

where $(\nabla \mathbf{V})^{T}$ is the transpose of $\nabla \mathbf{V}$.

Now substituting (1) and (5) in the constitutive equation (6) for the fourth grade fluid, we obtain the following physical components of the Cauchy stress tensor T:

$$
T_{x x}=-p+\alpha_{2}\left(\frac{d u}{d y}\right)^{2}-2 \beta_{2} V_{0} \frac{d u}{d y} \frac{d^{2} u}{d y^{2}}+2 \gamma_{2} V_{0}^{2} \frac{d u}{d y} \frac{d^{3} u}{d y^{3}}+\gamma_{3} V_{0}^{2}\left(\frac{d^{2} u}{d y^{2}}\right)^{2}+2 \gamma_{6}\left(\frac{d u}{d y}\right)^{4}
$$

$$
\begin{aligned}
T_{x y}=\mu \frac{d u}{d y} & -\alpha_{1} V_{0} \frac{d^{2} u}{d y^{2}}+\beta_{1} V_{0}^{2} \frac{d^{3} u}{d y^{3}}+2\left(\beta_{1}+\beta_{3}\right)\left(\frac{d u}{d y}\right)^{3}-\gamma_{1} V_{0}^{3} \frac{d^{4} u}{d y^{4}} \\
& -\left(6 \gamma_{2}+2 \gamma_{3}+2 \gamma_{4}+2 \gamma_{5}+6 \gamma_{7}+2 \gamma_{8}\right) V_{0}\left(\frac{d u}{d y}\right)^{2} \frac{d^{2} u}{d y^{2}}
\end{aligned}
$$

$$
\begin{aligned}
T_{y y}= & -p+\left(2 \alpha_{1}+\alpha_{2}\right)\left(\frac{d u}{d y}\right)^{2}-2 V_{0}\left(3 \beta_{1}+\beta_{2}\right) \frac{d u}{d y} \frac{d^{2} u}{d y^{2}}+6 \gamma_{1} V_{0}^{2} \frac{d}{d y}\left(\frac{d u}{d y} \frac{d^{2} u}{d y^{2}}\right) \\
& +2\left(\gamma_{1}+\gamma_{2}\right) V_{0}^{2} \frac{d^{3} u}{d y^{3}} \frac{d u}{d y}+\gamma_{3} V_{0}^{2}\left(\frac{d^{2} u}{d y^{2}}\right)^{2}+2\left(2 \gamma_{3}+2 \gamma_{4}+2 \gamma_{5}+\gamma_{6}\right)\left(\frac{d u}{d y}\right)^{4}
\end{aligned}
$$

$$
\begin{aligned}
& T_{z z}=-p \\
& T_{x z}=T_{z y}=0 \\
& T_{x y}=T_{y x}, \quad T_{x z}=T_{z x}, \quad T_{y z}=T_{z y}
\end{aligned}
$$

Now, the three scalar components of the momentum equation (3), in view of Eqn. (5), become: 


$$
\begin{aligned}
-\rho V_{0} \frac{d u}{d y} & =\frac{\partial T_{x x}}{\partial x}+\frac{\partial T_{x y}}{\partial y}+\frac{\partial T_{x z}}{\partial z}-\sigma B_{0}^{2} u \\
0 & =\frac{\partial T_{x y}}{\partial x}+\frac{\partial T_{y y}}{\partial y}+\frac{\partial T_{y z}}{\partial z}+\sigma B_{0}^{2} V_{0} \\
0 & =\frac{\partial T_{x z}}{\partial x}+\frac{\partial T_{y z}}{\partial y}+\frac{\partial T_{z z}}{\partial z}
\end{aligned}
$$

where $\sigma$ is the electrical conductivity.

Inserting the above stress components in Eqns. (15)- (17), we obtain:

$$
\begin{gathered}
\mu \frac{d^{2} u}{d y^{2}}+\rho V_{0} \frac{d u}{d y}-\alpha_{1} V_{0} \frac{d^{3} u}{d y^{3}}+\beta_{1} V_{0}^{2} \frac{d^{4} u}{d y^{4}}+6\left(\beta_{2}+\beta_{3}\right)\left(\frac{d u}{d y}\right)^{2} \frac{d^{2} u}{d y^{2}}-\gamma_{1} V_{0}^{3} \frac{d^{5} u}{d y^{5}} \\
-2\left(3 \gamma_{2}+\gamma_{3}+\gamma_{4}+\gamma_{5}+3 \gamma_{7}+\gamma_{8}\right) V_{0} \frac{d}{d y}\left[\left(\frac{d u}{d y}\right)^{2} \frac{d^{2} u}{d y^{2}}\right]-\sigma B_{0}^{2} u=\frac{\partial \hat{p}}{\partial x} \\
\sigma B_{0}^{2} V_{0}=\frac{\partial \hat{p}}{\partial y} \\
0=\frac{\partial \hat{p}}{\partial z}
\end{gathered}
$$

where the modified pressure $\hat{p}$ is is given by:

$$
\begin{aligned}
\hat{p}=-T_{y y}= & p-\left(2 \alpha_{1}+\alpha_{2}\right)\left(\frac{d u}{d y}\right)^{2}+2 V_{0}\left(3 \beta_{1}+\beta_{2}\right) \frac{d u}{d y} \frac{d^{2} u}{d y^{2}}-6 \gamma_{1} V_{0}^{2} \frac{d}{d y}\left(\frac{d u}{d y} \frac{d^{2} u}{d y^{2}}\right) \\
& -2\left(\gamma_{1}+\gamma_{2}\right) V_{0}^{2} \frac{d^{3} u}{d y^{3}} \frac{d u}{d y}-\gamma_{3} V_{0}^{2}\left(\frac{d^{2} u}{d y^{2}}\right)^{2}-2\left(2 \gamma_{3}+2 \gamma_{4}+2 \gamma_{5}+\gamma_{6}\right)\left(\frac{d u}{d y}\right)^{4}
\end{aligned}
$$

Eqn. (20) indicates that $\hat{p}$ is not a function of $z$. On eliminating the pressure gradient from (18) and (19) by cross differentiation, we finally obtain:

$$
\begin{aligned}
-\rho V_{0} \frac{d^{2} u}{d y^{2}}= & \mu \frac{d^{3} u}{d y^{3}}-\sigma B_{0}^{2} \frac{d u}{d y}-\alpha_{1} V_{0} \frac{d^{4} u}{d y^{4}}+\beta_{1} V_{0}^{2} \frac{d^{5} u}{d y^{5}}+6\left(\beta_{2}+\beta_{3}\right) \frac{d}{d y}\left[\left(\frac{d u}{d y}\right)^{2} \frac{d^{2} u}{d y^{2}}\right] \\
& -\gamma_{1} V_{0}^{3} \frac{d^{6} u}{d y^{6}}-2\left(3 \gamma_{2}+\gamma_{3}+\gamma_{4}+\gamma_{5}+3 \gamma_{7}+\gamma_{8}\right) V_{0} \frac{d^{2}}{d y^{2}}\left[\left(\frac{d u}{d y}\right)^{2} \frac{d^{2} u}{d y^{2}}\right]
\end{aligned}
$$


Equation (22) is the stationary form of the one used by Wang and $\mathrm{Wu}$ [10]. The appropriate partial slip boundary conditions are:

$$
\begin{aligned}
& u(0)=\left.\lambda_{1} T_{x y}\right|_{y=0} \\
& u(y) \rightarrow U_{\infty} \text { as } y \rightarrow \infty
\end{aligned}
$$

where $\lambda_{1}$ is the slip factor and $U_{\infty}$ is the mainstream velocity.

By introducing the following non-dimensional quantities, with $\nu$ the kinematic viscosity of the fluid,

$$
\begin{aligned}
& \bar{u}=\frac{u}{U_{\infty}}, \quad \bar{y}=\frac{U_{\infty} y}{\nu}, \quad \bar{V}_{0}=\frac{V_{0}}{U_{\infty}}, \\
& \bar{M}_{n}=\frac{\nu \sigma B_{0}^{2}}{\rho U_{\infty}^{2}}, \quad \bar{\alpha}_{1}=\frac{\alpha_{1} U_{\infty}^{2}}{\rho \nu^{2}}, \quad \bar{\beta}_{1}=\frac{\beta_{1} U_{\infty}^{4}}{\rho \nu^{3}}, \quad \bar{\gamma}_{1}=\frac{\gamma_{1} U_{\infty}^{6}}{\rho \nu^{4}}, \\
& \bar{\beta}=\frac{6\left(\beta_{2}+\beta_{3}\right) U_{\infty}^{4}}{\rho \nu^{3}}, \quad \bar{\gamma}=2\left(3 \gamma_{2}+\gamma_{3}+\gamma_{4}+\gamma_{5}+3 \gamma_{7}+\gamma_{8}\right) \frac{U_{\infty}^{6}}{\rho \nu^{4}}
\end{aligned}
$$

the boundary value problem (22) takes the form:

$$
\begin{aligned}
M_{n} \frac{d u}{d y}-V_{0} \frac{d^{2} u}{d y^{2}}-\frac{d^{3} u}{d y^{3}} & +\alpha_{1} V_{0} \frac{d^{4} u}{d y^{4}}-\beta_{1} V_{0}^{2} \frac{d^{5} u}{d y^{5}}+\gamma_{1} V_{0}^{3} \frac{d^{6} u}{d y^{6}}=\beta\left[2 \frac{d u}{d y}\left(\frac{d^{2} u}{d y^{2}}\right)^{2}\right. \\
& \left.+\left(\frac{d u}{d y}\right)^{2} \frac{d^{3} u}{d y^{3}}\right]-\gamma V_{0}\left[2\left(\frac{d^{2} u}{d y^{2}}\right)^{3}+6 \frac{d u}{d y} \frac{d^{2} u}{d y^{2}} \frac{d^{3} u}{d y^{3}}+\left(\frac{d u}{d y}\right)^{2} \frac{d^{4} u}{d y^{4}}\right]
\end{aligned}
$$

and the boundary conditions (23) become:

$$
\begin{aligned}
& u=\lambda\left[\frac{d u}{d y}-V_{0} \alpha_{1} \frac{d^{2} u}{d y^{2}}+V_{0}^{2} \beta_{1} \frac{d^{3} u}{d y^{3}}+\frac{\beta}{3}\left(\frac{d u}{d y}\right)^{3}-V_{0}^{3} \gamma_{1} \frac{d^{4} u}{d y^{4}}-V_{0} \gamma\left(\frac{d u}{d y}\right)^{2} \frac{d^{2} u}{d y^{2}}\right] \text { at } y=0 \\
& u \rightarrow 1 \quad \text { as } \quad y \rightarrow \infty
\end{aligned}
$$

where $\lambda$ is the non-dimensional slip factor indicating the relative importance of the partial slip, and for simplicity, the bars of the non-dimensional quantities have been omitted. $M_{n}$ is the magnetic parameter and $\alpha_{1}, \beta_{1}, \gamma_{1}, \beta$ and $\gamma$ are the non-Newtonian flow parameters. 


\subsection{Heat transfer analysis}

We now turn our attention to the energy equation subject to two different sets of boundary conditions. In the first instance, we assume that the plate is at a temperature higher than the fluid. For the second case, we assume that the plate is insulated. Neglecting the radiant heating, the energy equation can be written as:

$$
\rho c_{p} \frac{d \theta}{d t}=\kappa \nabla^{2} \theta+\mathbf{T} \cdot \nabla \mathbf{V}
$$

where $c_{p}$ is the mass specific heat $(J / K / k g)$ and $\kappa$ the thermal conductivity $\left(\mathrm{kg} \cdot \mathrm{m} / \mathrm{s}^{3} / \mathrm{K}\right)$. Since the velocity and temperature depend only on $y$, using Eqns. (1) and (2) in the above energy equation (27), we get:

$$
-\rho c_{p} V_{0} \frac{d \theta}{d y}=\kappa \frac{d^{2} \theta}{d y^{2}}+T_{x y} \frac{d u}{d y}
$$

The boundary conditions depend on the following two heating processes.

\subsubsection{Constant surface temperature}

In this case, the appropriate boundary conditions are:

$$
\begin{aligned}
& \theta(0)=\theta_{w}, \\
& \theta(y) \rightarrow \theta_{\infty} \quad \text { as } \quad y \rightarrow \infty
\end{aligned}
$$

where $\theta_{w}$ is the wall temperature and $\theta_{\infty}$ is the temperature of the ambient fluid.

Using the non-dimensional temperature $\bar{\theta}=\frac{\theta-\theta_{\infty}}{\theta_{w}-\theta_{\infty}}$, the non-dimensional quantities (24) and the expression for $T_{x y}$ from Eqn. (10) in the energy equation (28), we obtain the following non-dimensional highly non-linear differential equation: 


$$
\begin{aligned}
\frac{d^{2} \theta}{d y^{2}}+P_{r} V_{0} \frac{d \theta}{d y}+P_{r} E_{c}\left[\left(\frac{d u}{d y}\right)^{2}-\alpha_{1} V_{0} \frac{d^{2} u}{d y^{2}} \frac{d u}{d y}\right. & +\beta_{1} V_{0}^{2} \frac{d^{3} u}{d y^{3}} \frac{d u}{d y}+\beta\left(\frac{d u}{d y}\right)^{4} \\
& \left.-\gamma V_{0}\left(\frac{d u}{d y}\right)^{3} \frac{d^{2} u}{d y^{2}}-\gamma_{1} V_{0}^{3} \frac{d^{4} u}{d y^{4}} \frac{d u}{d y}\right]=0
\end{aligned}
$$

where $P_{r}=\frac{\mu c_{p}}{\kappa}$ is the Prandtl number and $E_{c}=\frac{U_{\infty}^{2}}{c_{p}\left(T_{w}-T_{\infty}\right)}$ is the Eckert number and bars have been suppressed.

The boundary conditions for $\theta$ are:

$$
\begin{aligned}
& \theta(0)=1, \\
& \theta \rightarrow 0 \quad \text { as } \quad y \rightarrow \infty
\end{aligned}
$$

\subsubsection{Insulated surface}

In this case, the relevant boundary conditions are:

$$
\begin{aligned}
& \left.\frac{d \theta}{d y}\right|_{y=0}=0, \\
& \theta(y) \rightarrow \theta_{\infty} \quad \text { as } \quad y \rightarrow \infty
\end{aligned}
$$

Again, using the non-dimensional temperature $\bar{g}=\frac{\theta-\theta_{\infty}}{\theta_{w}-\theta_{\infty}}$, the non-dimensional quantities (24) and the expression for $T_{x y}$ from Eqn. (10) in the energy equation (28), we obtain the following highly non-linear differential equation:

$$
\begin{aligned}
\frac{d^{2} g}{d y^{2}}+P_{r} V_{0} \frac{d g}{d y}+P_{r} E_{c}\left[\left(\frac{d u}{d y}\right)^{2}-\alpha_{1} V_{0} \frac{d^{2} u}{d y^{2}} \frac{d u}{d y}\right. & +\beta_{1} V_{0}^{2} \frac{d^{3} u}{d y^{3}} \frac{d u}{d y}+\beta\left(\frac{d u}{d y}\right)^{4} \\
& \left.-\gamma V_{0}\left(\frac{d u}{d y}\right)^{3} \frac{d^{2} u}{d y^{2}}-\gamma_{1} V_{0}^{3} \frac{d^{4} u}{d y^{4}} \frac{d u}{d y}\right]=0
\end{aligned}
$$

which is same as Eqn.(30) and all the other parameters are the same as before. Bars have been omitted. 
The boundary conditions for $g$ are:

$$
\begin{aligned}
& \left.\frac{d g}{d y}\right|_{y=0}=0, \\
& g \rightarrow 0 \text { as } \quad y \rightarrow \infty
\end{aligned}
$$

\section{Numerical solution of the problem}

The system of highly non-linear differential equations (25) and (30) subject to boundary conditions (26) and (31), and the system of equations (25) and (33) subject to boundary conditions (26) and (34) are solved using a shooting method along with the fourth order Runge-Kutta method for different moderate values of the flow and heat transfer parameters. The effective Broyden's technique has been adopted in order to improve the initial guesses and to satisfy the boundary conditions at infinity. Moreover, it eases the computations by avoiding to compute the Jacobian matrix in each integration step. In fact, the above system of differential equations are highly non-linear, whose order is much more higher than the available known boundary conditions, which debars the use of the finite difference method without augmenting asymptotic boundary conditions at infinity $[6,10]$. It is noteworthy to mention that the shooting technique in calculating process does not require the entire starting boundary conditions, instead it only needs to match with the other end of the boundary condition to add amendment to its starting boundary conditions. Thus, it can process higher order differential equation problem that lacks of sufficient boundary conditions. Despite of few shortcomings of the shooting method, we found the shooting method quite suitable and aesthetically pleasing for the present investigation.

The semi-infinite integration domain $y \in[0, \infty)$ is replaced by a finite domain $y \in\left[0, y_{\infty}\right)$. In fact, $y_{\infty}$ usually depends upon the physical parameters of the problem and its value needs to be adjusted as the values of the parameters change. In practice, $y_{\infty}$ should be chosen sufficiently large so that the numerical solution closely approximates the terminal boundary conditions at $\infty$. Here, $y_{\infty}$ has been fixed to 10 .

The above systems of differential equations are converted into an equivalent initial value problem in order to use the fourth order Runge-Kutta method. For the sake of brevity, here we have explained the solution procedure for the system (25) and (30) subject to boundary conditions (26) and (31) concisely. The solution procedure for the system (25) and (33) subject to boundary conditions (26) and (34) is just similar. 
To reduce the equations to equivalent first order system, we set:

$$
\begin{aligned}
& u=z_{1}, \quad \frac{d u}{d y}=z_{2}, \quad \frac{d^{2} u}{d y^{2}}=z_{3}, \quad \frac{d^{3} u}{d y^{3}}=z_{4}, \quad \frac{d^{4} u}{d y^{4}}=z_{5}, \quad \frac{d^{5} u}{d y^{5}}=z_{6}, \\
& \theta=z_{7}, \quad \frac{d \theta}{d y}=z_{8},
\end{aligned}
$$

to finally get:

$$
\begin{aligned}
z_{1}^{\prime}=z_{2}, \quad z_{1}(0)=\lambda\left[s_{1}-\alpha_{1} V_{0} s_{2}+\beta_{1} V_{0}^{2} s_{3}+\frac{\beta}{3} s_{1}^{3}-\gamma_{1} V_{0}^{3} s_{4}-\gamma V_{0} s_{1}^{2} s_{2}\right] \\
z_{2}^{\prime}=z_{3}, \quad z_{2}(0)=s_{1} \\
z_{3}^{\prime}=z_{4}, \quad z_{3}(0)=s_{2} \\
z_{4}^{\prime}=z_{5}, \quad z_{4}(0)=s_{3} \\
z_{5}^{\prime}=z_{6}, \quad z_{5}(0)=s_{4} \\
z_{6}^{\prime}=-M_{n} z_{2}+V_{0} z_{3}+z_{4}-\alpha_{1} V_{0} z_{5}+\beta_{1} V_{0}^{2} z_{6}+\beta\left[2 z_{2} z_{3}^{2}+z_{2}^{2} z_{4}\right] \\
\quad-\gamma V_{0}\left[2 z_{3}^{3}+6 z_{2} z_{3} z_{4}+z_{2}^{2} z_{5}\right], \quad z_{6}(0)=s_{5} \\
z_{7}^{\prime}=z_{8}, \quad z_{7}(0)=1 \\
z_{8}^{\prime}=-P_{r} V_{0} z_{8}-P_{r} E_{c}\left[z_{2}^{2}-\alpha_{1} V_{0} z_{3} z_{2}+\beta_{1} V_{0}^{2} z_{4} z_{2}+\beta z_{2}^{4}-\gamma V_{0} z_{2}^{3} z_{3}-\gamma_{1} V_{0}^{3} z_{2} z_{5}\right], \\
\quad z_{8}(0)=s_{6} .
\end{aligned}
$$

Here, the prime indicates differentiation with respect to $y$. The essence of this method is to reduce the boundary value problem to an equivalent initial value problem and then use a shooting numerical technique to guess $s_{i}, \quad i=1,2, \ldots 6$, until the terminal boundary conditions $z_{1}=1, z_{7}=0$ are satisfied at $y=y_{\infty}$. Because of the non-linearities, which appear in the differential equations, the solution by the above method has been iterated and with reasonably close initial guesses to start the iterations, the convergence to the actual values within an accuracy of $O\left(10^{-6}\right)$ is attained after only 9 to 11 iterations.

\section{Results and discussion}

Keeping in view of a proliferation of the number of parameters, we have restricted the discussions of the obtained results to the effects of emerging important flow parameters $\alpha_{1}, \beta, \gamma, V_{0}, M_{n}, P_{r}, E_{c}$, and the newly introduced slip parameter $\lambda$ on the velocity and temperature fields. It is found that $\beta, \beta_{1}$, and similarly $\gamma, \gamma_{1}$ have similar effects on the velocity and temperature fields. Further, one can refer the works of Hayat et al. [6,9] and Sajid et al. [8] for a precise discussion with the conventional no-slip boundary conditions. 
As a preliminary test [16] to check the accuracy of the solution, it may be noted that for the no-slip and non magnetic case, at $y=0$, Eqn. (25) reduces to:

$$
\begin{aligned}
V_{0} \frac{d^{2} u}{d y^{2}}-\frac{d^{3} u}{d y^{3}} & +\alpha_{1} V_{0} \frac{d^{4} u}{d y^{4}}-\beta_{1} V_{0}^{2} \frac{d^{5} u}{d y^{5}}+\gamma_{1} V_{0}^{3} \frac{d^{6} u}{d y^{6}}-\beta\left[2 \frac{d u}{d y}\left(\frac{d^{2} u}{d y^{2}}\right)^{2}\right. \\
& \left.+\left(\frac{d u}{d y}\right)^{2} \frac{d^{3} u}{d y^{3}}\right]+\left.\gamma V_{0}\left[2\left(\frac{d^{2} u}{d y^{2}}\right)^{3}+6 \frac{d u}{d y} \frac{d^{2} u}{d y^{2}} \frac{d^{3} u}{d y^{3}}+\left(\frac{d u}{d y}\right)^{2} \frac{d^{4} u}{d y^{4}}\right]\right|_{y=0}=0
\end{aligned}
$$

For all solutions computed for different ranges of the flow parameters, the left hand side of Eqn. (37) is found to be less than $10^{-4}$.

The variations of the non-dimensional velocity $u$ with different flow parameters have been plotted in Figs. 2-7 for $\beta_{1}=1$ and $\gamma_{1}=1$. Fig. 2 depicts that in presence of slip, as the normal stress coefficient $\alpha_{1}$ increases from zero, the velocity increases near the surface and then starts decreasing away from the surface of the plate, resulting in a 'cross-over' in the profile. It is noteworthy to mention that such a cross-over is absent for the no-slip case [9]. Figs. 3 and 4 show that velocity decreases, or in other words, the momentum boundary layer thickness increases with an increase in the non-Newtonian fluid parameters $\beta$ and $\gamma$ respectively. These findings are in agreement with those reported by Hayat et al. [9] for the flow past a porous flat plate or by Islam et al. [3] for a plane Poiseuille flow. In general, the fourth grade non-Newtonian fluid exhibits a thicker boundary layer than the Newtonian fluid, and increasing the higher order material parameters of non-Newtonian fluid causes further thickening of the boundary layer. Fig. 5 shows the variation of $u$ with the nondimensional suction velocity. It is again interesting to find that in presence of slip, as the suction increases, the velocity near the plate increases, and then decreases gradually. This results in a cross-over in the velocity profile. However, in absence of slip, such a cross-over in not found, as reported by Sajid et al. [8] and Hayat et al. [9]. Fig. 6 reveals that an increase in the magnetic interaction parameter $M_{n}$ increases the velocity throughout the domain of integration. The effect of slip on the velocity profile, when other flow parameters are kept constant, can be seen from Fig. 7. As was expected, an increase in slip results in an increase in the velocity near the surface of the plate. The velocity approaches towards the free stream value 1 with an increase in the slip, and flow behaves as though it were inviscid.

Another interesting quantity is the skin-friction coefficient at the wall. It is related to the wall shear stress, given by the Eqn. (10), which in the dimensionless form becomes: 
$\left.T_{x y}\right|_{y=0}=\left[\frac{d u}{d y}-V_{0} \alpha_{1} \frac{d^{2} u}{d y^{2}}+V_{0}^{2} \beta_{1} \frac{d^{3} u}{d y^{3}}+\frac{\beta}{3}\left(\frac{d u}{d y}\right)^{3}-V_{0}^{3} \gamma_{1} \frac{d^{4} u}{d y^{4}}-V_{0} \gamma\left(\frac{d u}{d y}\right)^{2} \frac{d^{2} u}{d y^{2}}\right]_{y=0}$

The effect of the fourth grade fluid parameter $\gamma$ on the non-dimensional wall shear stress $\left.T_{x y}\right|_{y=0}$ is clear from Fig 8 . It shows that $\left.T_{x y}\right|_{y=0}$ increases, resulting in an increase in the momentum boundary layer thickness, with an increase in $\gamma$. On the other hand, as was expected, $\left.T_{x y}\right|_{y=0}$ decreases rapidly with an increase in the slip parameter $\lambda$ (Fig 9), indicating a substantial decrease in the momentum boundary layer thickness.

Figs. 10-21 indicate the effects of different emerging flow parameters on the non-dimensional temperature profiles $\theta(y)$ and $g(y)$ for the constant surface temperature and the insulated wall cases, when $\beta_{1}=\gamma_{1}=1, P_{r}=3$, and $E_{c}=0.5$. Figs 10-12 respectively depict that even in presence of slip, an increase in the non-Newtonian parameters $\alpha_{1}, \beta$ and $\gamma$ increase the temperature $\theta(y)$. These findings are in agreement with those reported by Hayat et al. [9]. The effect of the fourth garde fluid parameter $\gamma$ on $\theta$ is not prominent throughout the domain of integration, as shown in Fig. 12. Figs. 13-15 show that the temperature, and hence the thermal boundary layer thickness, decreases with an increase in the suction, magnetic parameter and slip parameter respectively. The suction has a prominent effect on the temperature profile. Figs. 16-21 depict the effects of various flow parameters on the non-dimensional temperature $g(y)$ for the insulated wall case. It is clear that all the flow parameters have similar effects of $\theta(y)$ and $g(y)$. Fig. 21 shows that the slip has a substantial effect on the thermal boundary layer for the insulated wall case.

The dimensionless surface temperature gradient $\theta^{\prime}(0)$ in the constant surface temperature case, and the dimensionless surface temperature $g(0)$ in the insulated wall case against different flow parameters have been tabulated in Table 1. It is clear that the shear thickening parameter $\beta$ decreases the magnitude of the rate of heat transfer $\theta^{\prime}(0)$, when the other parameters are kept constant. It can be of interest to find that the heat transfer rate increases with $\gamma$ in presence of comparatively large value $(\lambda=2)$ of the slip parameter. The slip parameter $\lambda$ increase the rate of heat transfer at the surface of the plate. Moreover, it is observed that for constant $E_{c}$, the value of $\left|\theta^{\prime}(0)\right|$ increases with $P_{r}$, since the thermal boundary layer thickness $\delta_{T}$ decreases with $P_{r}$ due to the estimate $\delta_{T} \sim \frac{1}{\sqrt{P_{r}}}$. Apparently, the surface temperature $g(0)$ in the insulated wall case increases with $E_{c}$, when other flow parameters are kept constant. 


\section{Conclusions}

Flow and heat transfer of an electrically conducting fourth grade fluid past an infinite plate with partial slip boundary condition, subject to uniform suction has been considered in this paper. Due to the presence of higher order material derivatives of the strain tensor in the constitutive equation of the fourth grade fluid, the momentum equation gives rise to a highly nonlinear differential equation of order six with only two adherence boundary conditions. The resulting system of differential equations with inadequate boundary conditions has been solved by the shooting method along with the fourth order Runge-Kutta method and the Broyden's method. The use of the Broyden's method as a zero-finding algorithm has enhanced the efficiency of the shooting technique by reducing the computational time. The combined effects of the emerging non-Newtonian flow parameters and slip on the momentum and thermal boundary layers have been discussed elaborately and shown graphically. It is interesting to find the exceptional cross-overs in the velocity profile in the presence of slip. The fourth grade fluid parameter $\gamma$ increases the momentum boundary layer thickness, whereas the slip parameter substantially decreases it. Similarly, the non-Newtonian fourth grade fluid parameters and the slip have opposite effects on the thermal boundary layer thickness.

The present study will be extended in a close future to take into account additional effects such as the dependance of the slip velocity on the normal stress, Joule heating in the energy equation or the thermal slip condition. As the shooting method limited the present investigation to moderate values of the flow parameters, a more effective and suitable numerical technique would be also suitable to see the effects of higher values of the fourth grade and slip parameters on the Blasius flow problem.

\section{References}

[1] M. Renardy. On the domain space for constitutive laws in linear viscoelasticity. Arch. Rat. Mech. Anal., 85(1):21-26, 1984.

[2] T. Hayat, R. Naz, and S. Abbasbandy. On flow of a fourth-grade flud with heat transfer. Int. J. Numer. Meth. Fluids, (67):2043-2053, 2011.

[3] S. Islam, Z. Bano, I. Siddique, and A.M. Siddiqui. The optimal solution for the flow of a fourth-grade fluid with partial slip. Comput. Math. Applic., 61:1507$1516,2011$.

[4] R.A. Shah, S. Islam, and A.M. Siddiqui. Couette and Poiseuille flows for fourth grade fluids using optimal homotopy asymptotic method. World Appl. Sci. J., 9(11):1228-1236, 2010. 
[5] O.U. Mehmood, N. Mustapha, and S. Shafie. Heat transfer on peristaltic flow of fourth grade fluid in inclined asymmetric channel with partial slip. Appl. Math. Mech. - Engl. Ed., DOI: 10.1007/s10483-012-1624-6.

[6] T. Hayat, Y. Wang, and K. Hutter. Flow of a fourth grade fluid. Math. Models Meth. Appl. Sci., 12(6):797-811, 2002.

[7] T. Hayat, A.H. Kara, and E. Momoniat. The unsteady flow of a fourth-grade fluid past a porous plate. Math. Comp. Model., 41:1347-1353, 2005.

[8] M. Sajid, T. Hayat, and S. Asghar. On the analytic solution of the steady flow of a fourth grade fluid. Phys. Letters A, 335(1):18-26, 2006.

[9] T. Hayat, S. Noreen, and M. Sajid. Heat transfer analysis of the steady flow of a fourth grade fluid. Int. J. Thermal Sci., 47(5):591-599, 2008.

[10] Y. Wang and W. Wu. Unsteady flow of a fourth grade fluid due to an oscillating plate. Int. J. Non-Linear Mech., 42(3):432-441, 2007.

[11] V. Marinca, N. Herisanu, C. Bota, and B. Marinca. An optimal homotopy asymptotic method applied to the steady flow of a fourth-grade fluid past a porous plate. Appl. Maths. Letter, 22:245-251, 2009.

[12] T. Hayat, M. Asif Farooq, T. Javed, and M. Sajid. Partial slip effects on the flow and heat transfer characteristics in a third grade fluid. Nonlinear Analysis: Real World Applications, 10(2):745-755, 2009.

[13] S. Nadeem, T. Hayat, S. Abbasbandy, and M. Ali. Effects of partial slip on a fourth-grade fluid with variable viscosity: An analytic solution. Nonlinear Analysis: Real World Applications, 11(2):856-868, 2010.

[14] B. Sahoo. Flow and heat transfer of an electrically conducting third grade fluid past an infinite plate with partial slip. Meccanica, 45(3):319-330, 2010.

[15] J.A. Shercliff. A text book of magnetohydrodynamics. Pergamon Press, Oxford, 1965.

[16] V.K. Garg and K.R. Rajagopal. Stagnation point flow of a non-Newtonian fluid. Mech. Res. Comm., 17:415-421, 1990. 


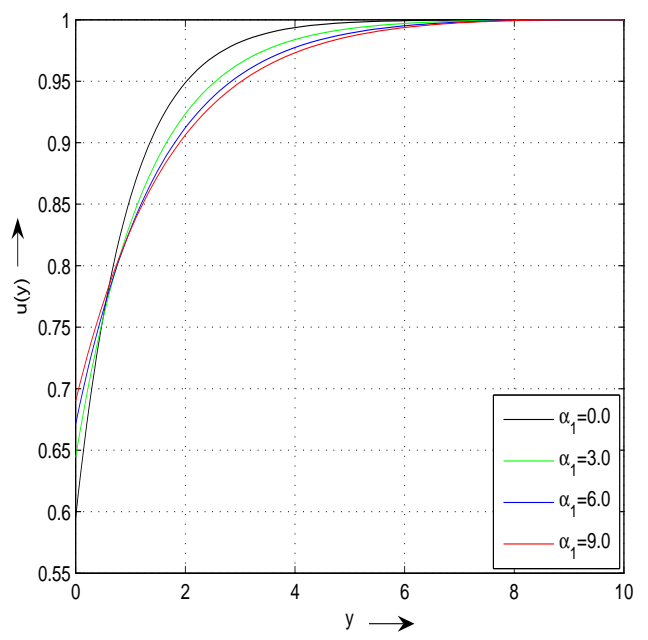

Fig. 2. Variation of $u$ with $\alpha_{1}$ at $\beta=2.0, \gamma=1.0, V_{0}=0.5$, $M_{n}=1.0 \& \lambda=1.0$.

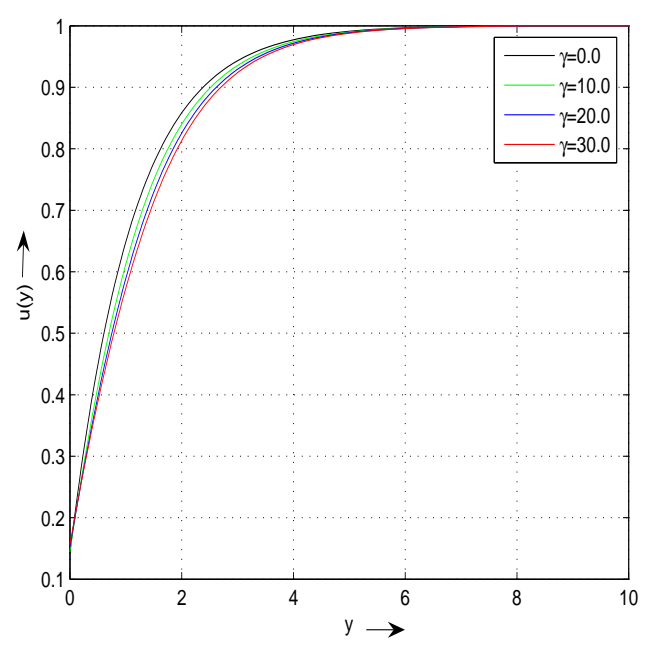

Fig. 4. Variation of $u$ with $\gamma$ at $\alpha_{1}=1.0, \beta=2.0, V_{0}=0.5$, $M_{n}=1.0 \& \lambda=0.1$.

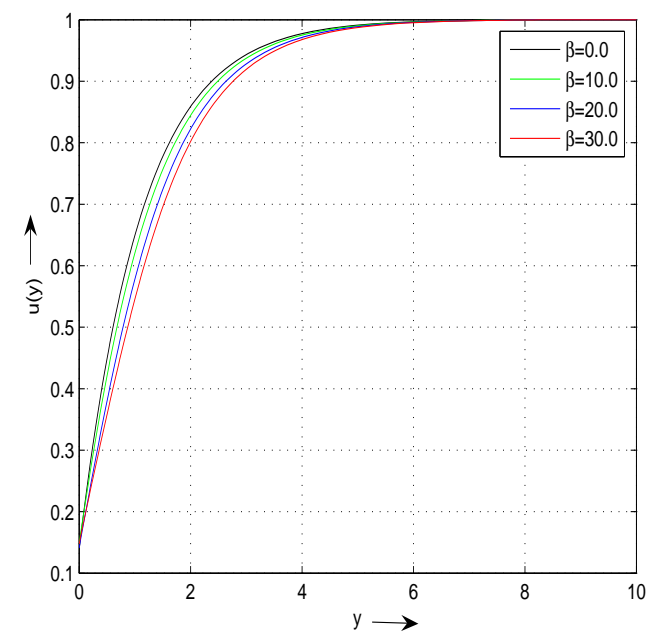

Fig. 3. Variation of $u$ with $\beta$ at $\alpha_{1}=1.0, \gamma=1.0, V_{0}=0.5$, $M_{n}=1.0 \& \lambda=0.1$.

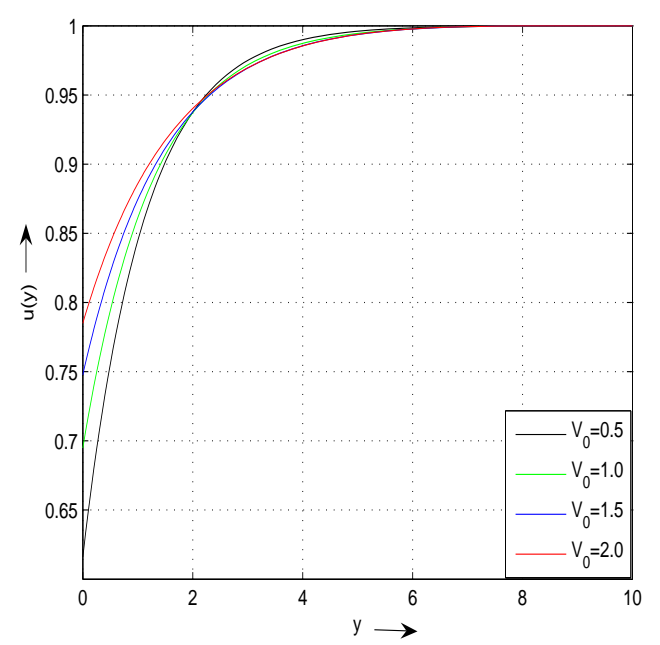

Fig. 5. Variation of $u$ with $V_{0}$ at $\alpha_{1}=1.0, \beta=2.0, \gamma=1.0$, $M_{n}=1.0 \& \lambda=1.0$. 


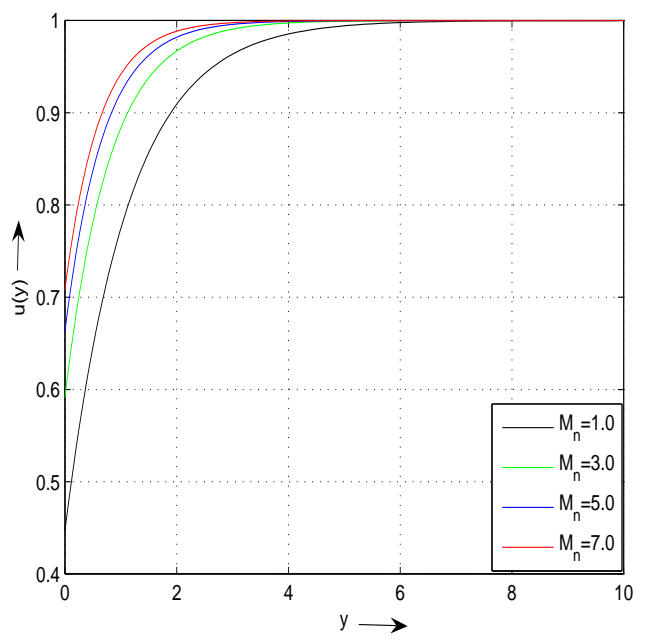

Fig. 6. Variation of $u$ with $M_{n}$ at $\alpha_{1}=1, \beta=2, \gamma=1, V_{0}=0.5 \&$ $\lambda=0.5$.

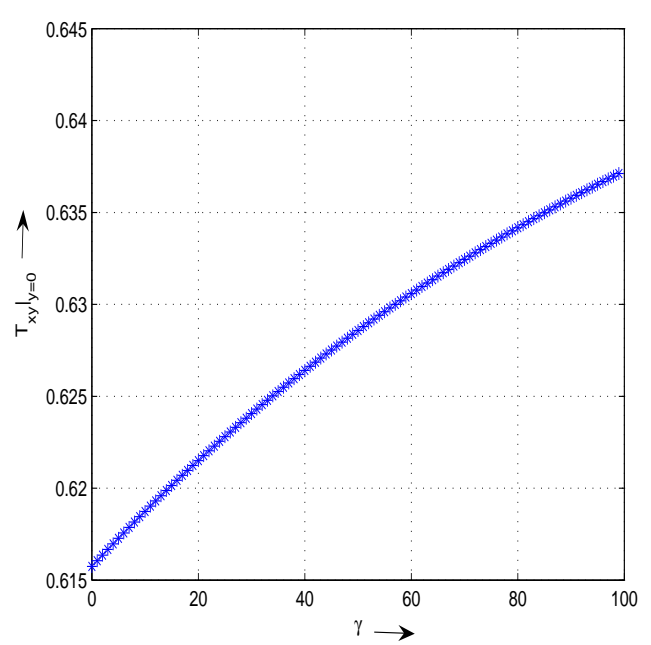

Fig. 8. Variation of $\left.T_{x y}\right|_{y=0}$ with $\gamma$ at $\alpha_{1}=1, \beta=2, V_{0}=0.5, M_{n}=1$ $\& \lambda=1$.

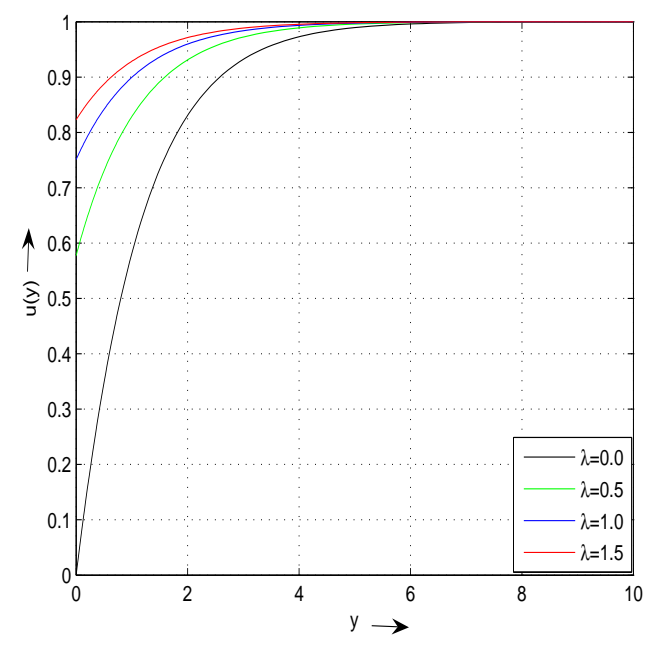

Fig. 7. Variation of $u$ with $\lambda$ at $\alpha_{1}=1, \beta=2, \gamma=1, V_{0}=0.5$ $\& M_{n}=1$.

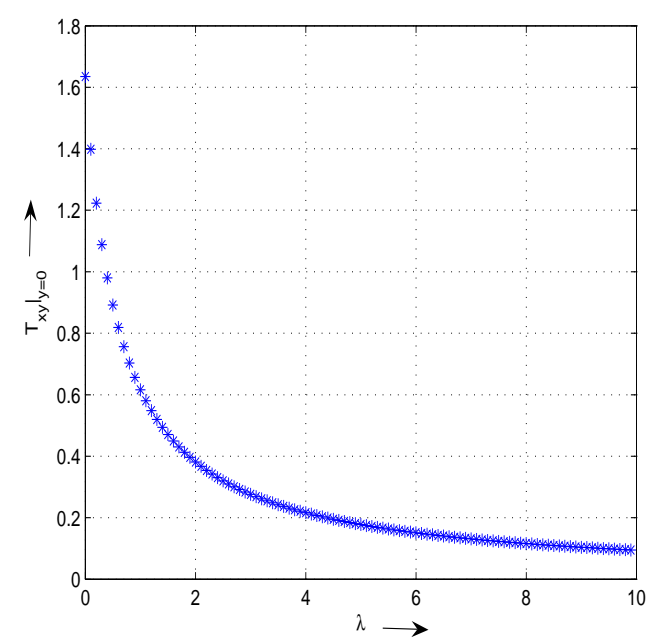

Fig. 9. Variation of $\left.T_{x y}\right|_{y=0}$ with $\lambda$ at $\alpha_{1}=1, \beta=2, \gamma=1, V_{0}=0.5 \&$ $M_{n}=1$. 


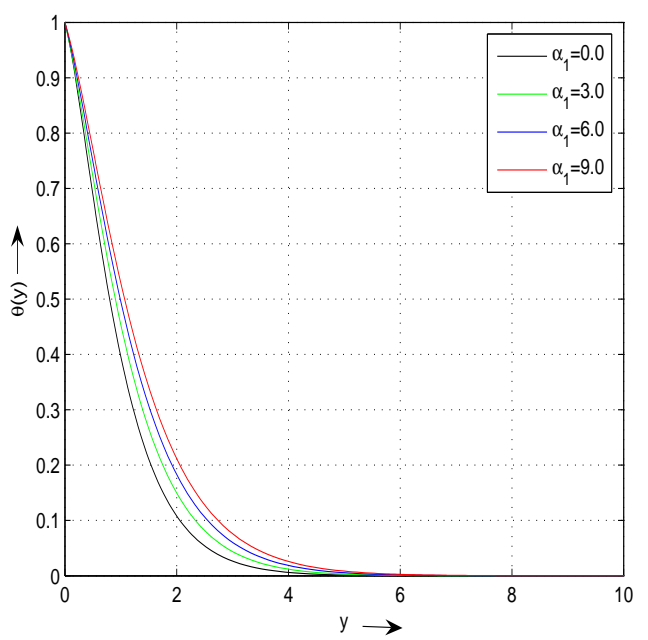

Fig. 10. Variation of $\theta$ with $\alpha_{1}$ at $\beta=2, \gamma=1, V_{0}=0.5, M_{n}=1 \&$ $\lambda=0.1$.

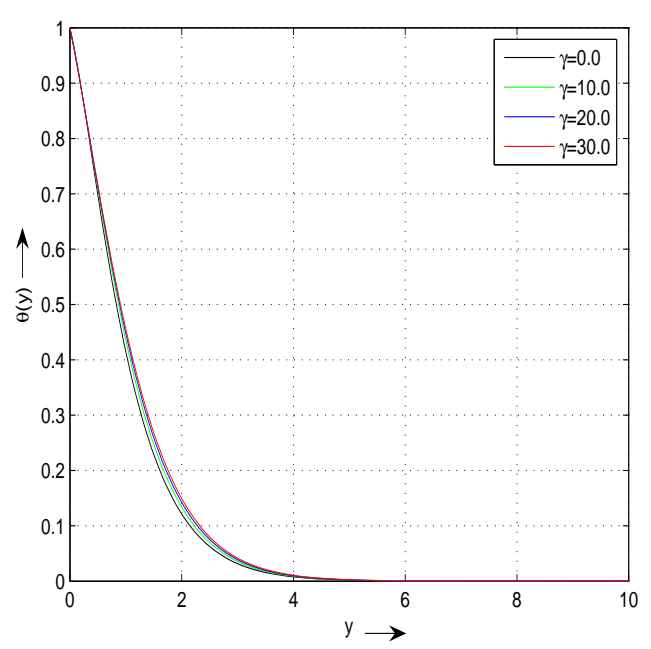

Fig. 12. Variation of $\theta$ with $\gamma$ at $\alpha_{1}=1, \beta=2, V_{0}=0.5, M_{n}=1$ $\& \lambda=0.1$.

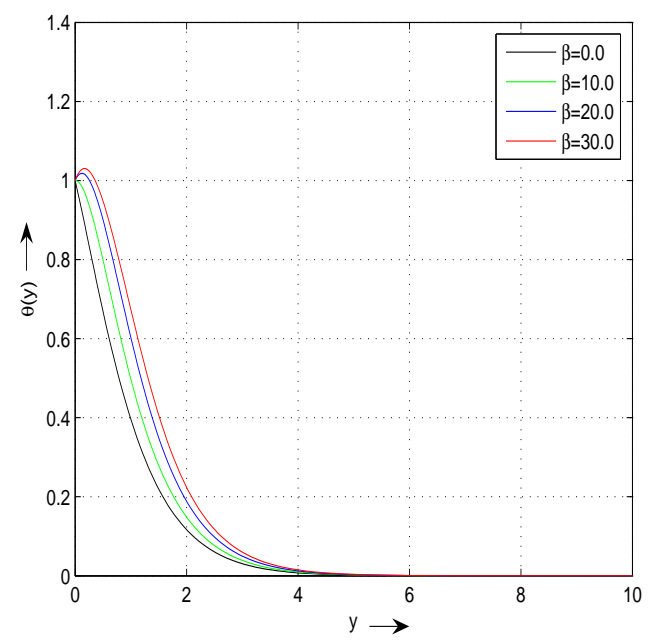

Fig. 11. Variation of $\theta$ with $\beta$ at $\alpha_{1}=1, \gamma=1, V_{0}=0.5, M_{n}=1$ $\& \lambda=0.1$.

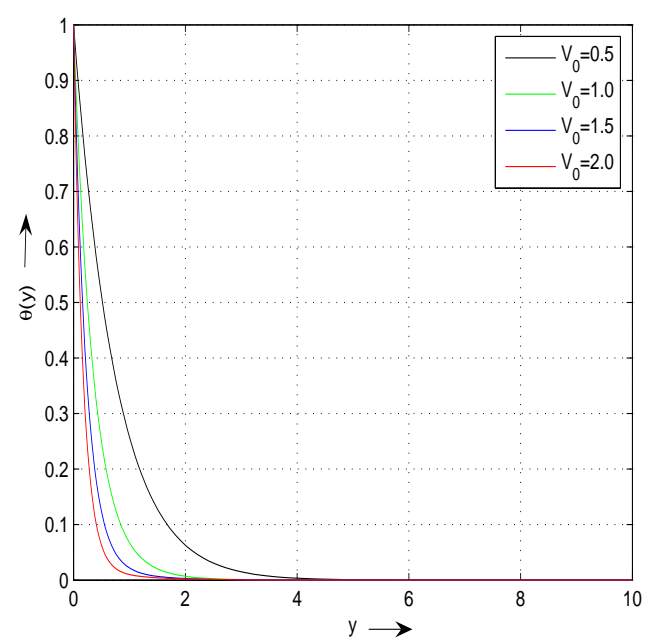

Fig. 13. Variation of $\theta$ with $V_{0}$ at $\alpha_{1}=1, \beta=2, \gamma=1, M_{n}=1 \&$ $\lambda=1$. 


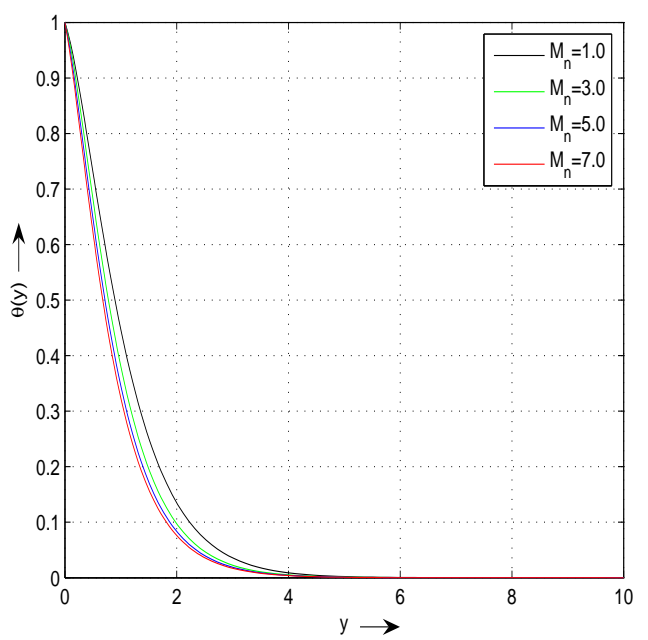

Fig. 14. Variation of $\theta$ with $M_{n}$ at $\alpha_{1}=1, \beta=2, \gamma=1, V_{0}=0.5 \&$ $\lambda=0.5$.

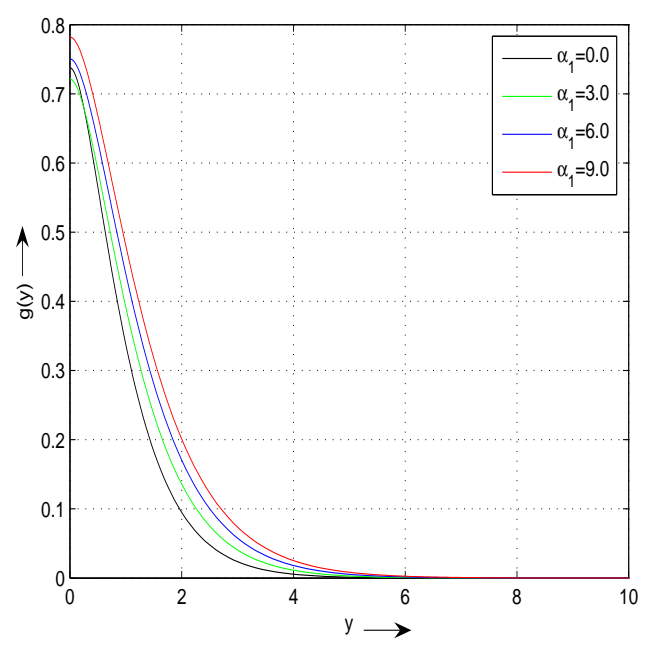

Fig. 16. Variation of $g$ with $\alpha_{1}$ at $\beta=2, \gamma=1, V_{0}=0.5, M_{n}=1 \&$ $\lambda=0.1$.

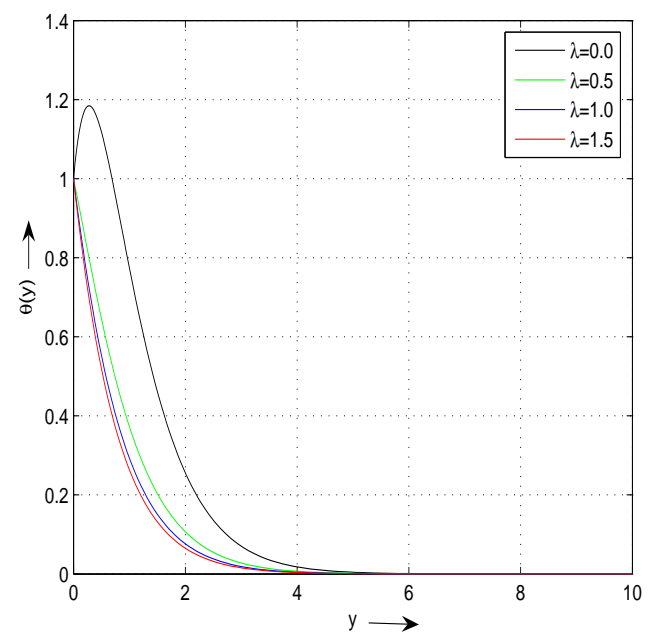

Fig. 15. Variation of $\theta$ with $\lambda$ at $\alpha_{1}=1, \beta=2, \gamma=1, V_{0}=0.5$ $\& M_{n}=1$.

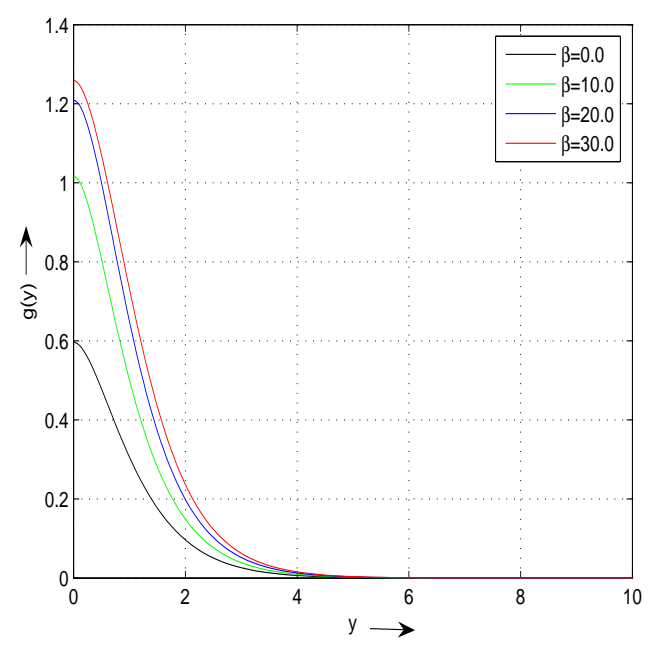

Fig. 17. Variation of $g$ with $\beta$ at $\alpha_{1}=1, \gamma=1, V_{0}=0.5, M_{n}=1$ $\& \lambda=0.1$. 


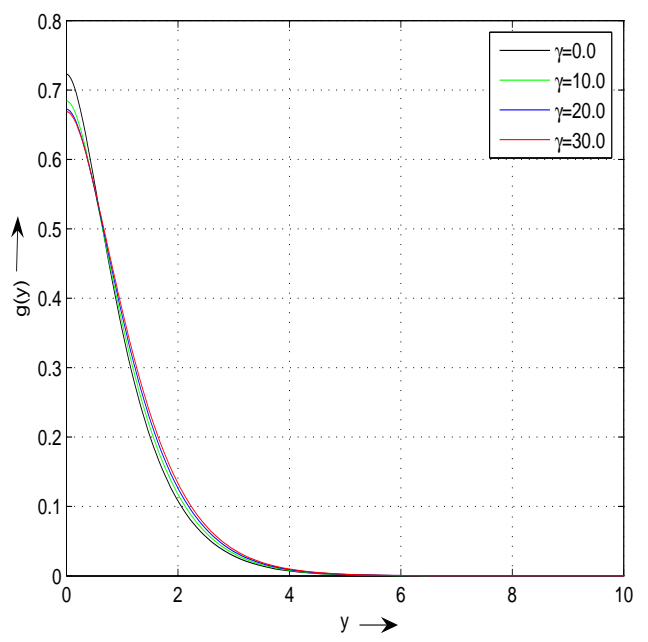

Fig. 18. Variation of $g$ with $\gamma$ at $\alpha_{1}=1, \beta=2, V_{0}=0.5, M_{n}=1$ $\& \lambda=0.1$.

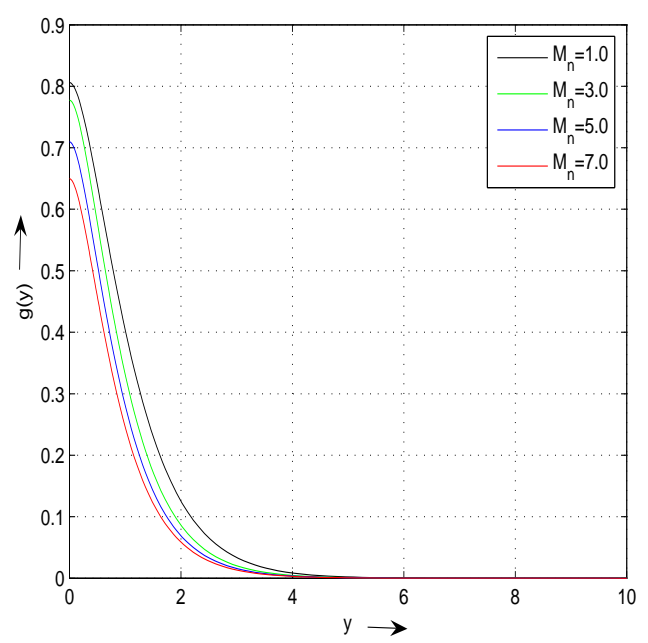

Fig. 20. Variation of $g$ with $M_{n}$ at $\alpha_{1}=1, \beta=2, \gamma=1, V_{0}=0.5 \&$ $\lambda=0.5$.

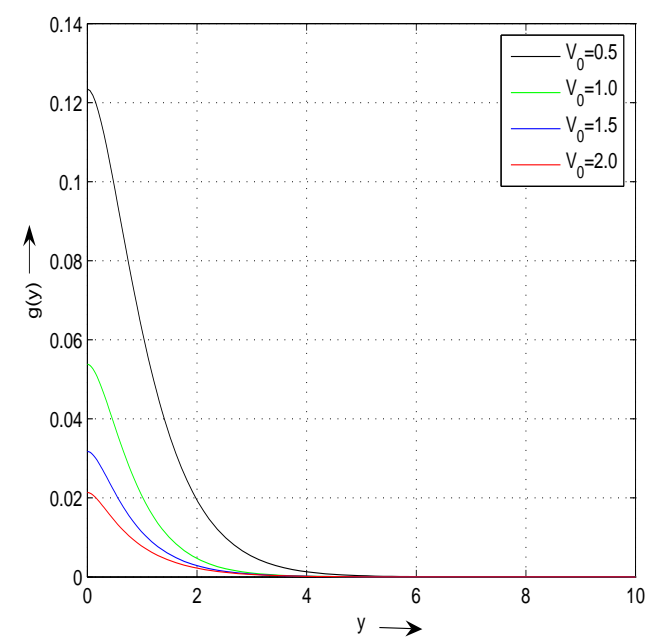

Fig. 19. Variation of $g$ with $V_{0}$ at $\alpha_{1}=1, \beta=2, \gamma=1, M_{n}=1 \&$ $\lambda=1$.

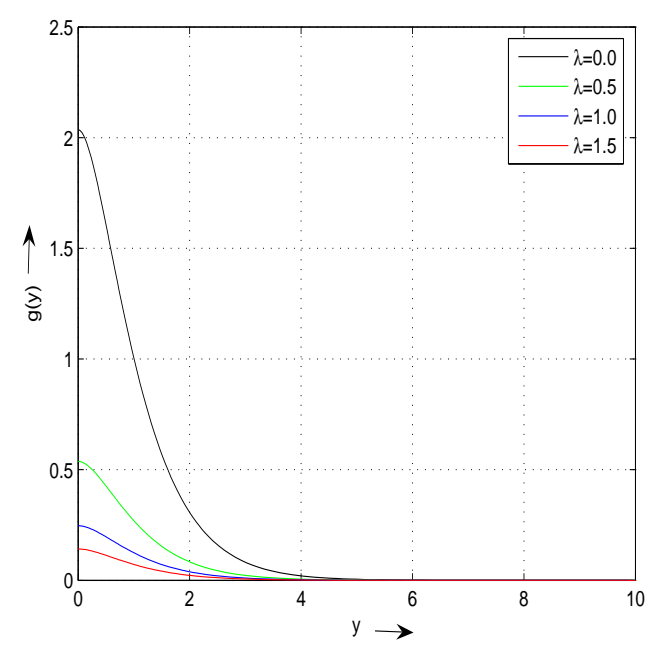

Fig. 21. Variation of $g$ with $\lambda$ at $\alpha_{1}=1, \beta=2, \gamma=1, V_{0}=0.5$ $\& M_{n}=1$. 


\begin{tabular}{|c|c|c|c|c|c|c|c|c|c|}
\hline$\alpha_{1}$ & $\beta$ & $\gamma$ & $V_{0}$ & $M_{n}$ & $\lambda$ & $P_{r}$ & $E_{c}$ & $\theta^{\prime}(0)$ & $g(0)$ \\
\hline 0.1 & & & & & & & & -3.826486 & 0.043378 \\
\hline 0.3 & 1.0 & 2.0 & 2.0 & 1.0 & 1.0 & 2.0 & 1.0 & -3.827067 & 0.043233 \\
\hline \multirow[t]{2}{*}{0.5} & & & & & & & & -3.827638 & 0.043090 \\
\hline & 0.5 & & & & & & & -2.840175 & 0.053275 \\
\hline \multirow[t]{3}{*}{1.0} & 1.5 & 2.0 & 1.0 & 1.0 & 1.0 & 3.0 & 0.5 & -2.839138 & 0.053621 \\
\hline & 2.5 & & & & & & & -2.838103 & 0.053966 \\
\hline & & 1.0 & & & & & & -0.490230 & 0.026147 \\
\hline \multirow[t]{3}{*}{2.0} & 1.0 & 1.5 & 0.5 & 1.0 & 2.0 & 1.0 & 0.3 & -0.490232 & 0.026142 \\
\hline & & 2.0 & & & & & & -0.490235 & 0.026137 \\
\hline & & & 1.0 & & & & & -0.492547 & 0.021544 \\
\hline \multirow[t]{3}{*}{3.0} & 2.0 & 1.0 & 3.0 & 0.5 & 1.0 & 3.0 & 0.5 & -0.503001 & 0.000777 \\
\hline & & & 5.0 & & & & & -0.503356 & 0.000071 \\
\hline & & & & 0.1 & & & & -0.935282 & 0.064760 \\
\hline \multirow[t]{3}{*}{3.0} & 1.0 & 2.0 & 1.0 & 0.3 & 1.0 & 3.0 & 0.5 & -0.969405 & 0.030639 \\
\hline & & & & 0.5 & & & & -0.969405 & 0.030639 \\
\hline & & & & & 1.0 & & & -2.848080 & 0.050640 \\
\hline \multirow[t]{3}{*}{4.0} & 2.0 & 2.0 & 1.0 & 1.0 & 2.0 & 3.0 & 0.5 & -2.949012 & 0.016996 \\
\hline & & & & & 3.0 & & & -2.974725 & 0.008425 \\
\hline & & & & & & 1.0 & & -1.958481 & 0.020760 \\
\hline \multirow[t]{3}{*}{3.0} & 1.0 & 2.0 & 2.0 & 1.0 & 1.0 & 2.0 & 0.5 & -3.916961 & 0.020759 \\
\hline & & & & & & 3.0 & & -5.875442 & 0.020757 \\
\hline & & & & & & & 0.5 & -3.973168 & 0.006708 \\
\hline \multirow[t]{2}{*}{1.0} & 3.0 & 2.0 & 2.0 & 1.0 & 2.0 & 2.0 & 1.5 & -3.919504 & 0.020124 \\
\hline & & & & & & & 2.0 & -3.865840 & 0.033540 \\
\hline
\end{tabular}

Table $\overline{\overline{1}}$

Variations of $\theta^{\prime}(0)$ and $g(0)$ with different flow parameters. 